\title{
Causation and Melanoma Classification
}

Philosophy of Medicine Roundtable

October 19-20, 2009

Erasmus Institute for Philosophy and Economics (EIPE)

Rotterdam, the Netherlands

\author{
Brendan Clarke \\ PhD Candidate \\ Department of Science and Technology Studies, UCL \\ Gower Street \\ London, WC1E 6BT \\ b.clarke@ucl.ac.uk
}

\begin{abstract}
While the clinical syndrome of melanoma was described during the nineteenth century, the pathology, aetiology and classification of the tumour remained controversial well into the twentieth. For instance, the debate about the type of tumour, its aetiology, classification and the relationship between pigmented moles and melanoma were common. This debate was widely regarded as settled by the 1960s, and we are now accustomed to think of melanoma as a single pathological and aetiological entity, with a number of possible clinical manifestations. A number of systems of classification are in common use. We may classify by site. So melanoma may occur on the skin (cutaneous), on the palms, soles and under the nails (acral) or on mucosal surfaces (mucosal). We may classify by tumour morphology - so tumours may be superficial spreading or nodular. Or we may classify by combinations of site and morphology.
\end{abstract}

There are problems for these unificatory classifications, however. For instance, certain types of tumour (acral in particular) have widely varying incidence by race. A more significant challenge arises from recent research findings, especially the discovery that various genetic mutations (notably c-KIT and BRAF) seem to correlate with particular sub-types of melanoma. Their aetiological role is supported by the therapeutic success of agents directed against them. This suggests that the conventional systems of classification are in trouble, and much ongoing work is directed toward finding new ways of differentiating melanoma types with the intention of improving clinical outcomes, which will potentially require the clinical management of melanoma subtypes as distinct diseases.

This raises exciting philosophical issues. For instance, what are the implications for systematic disease classification? Is there an optimum best single system for cases like these, or can we legitimately be more pluralistic, with possibly overlapping classificatory systems? How about the role of unification in this case? There is much debate to be found in the historical melanoma literature regarding the relative wisdom of either "lumping" and "splitting" as methodological approaches, with the repeated suggestion that strong forms of either strategies seemed likely to lead to difficulties.

There is much to discuss here. However, I will focus on one area in particular - causation - with two aims. First, to outline the historical role of causation in the development of melanoma classification. Second, to suggest that, owing to the exploitable features of causal relationships, an attempt at a causal classification may be the optimal strategy in this case. 


\section{Introduction}

What's the point of this paper? It is to suggest, first, that disease classification should not be solely the concern of the philosopher or medical researcher, but is of real clinical importance in day to day medical practice. I'll outline how current melanoma classifications are deficient for such clinical purposes, and suggest that these failures result from a lack of consideration of causal factors. Finally, I'll suggest that a new group of classifications which make use of causal thinking appear likely to resolve some of these empirical problems, and I'll make some philosophical remarks detailing why I feel entitled to be optimistic about them.

To do this, I'll give a brief history of melanoma, and describe some attempts at classification. I'll then move on to discuss intimate relationship between causation, classification and the methodology of biomedical research programmes.

\section{§1: Melanoma causation}

So what is melanoma? It is a common malignant tumour of the melanin-containing cells of the skin. Its incidence varies geographically, from 5 to 40 cases per 100000 population per year [Marks, 2000: 460]. This incidence is steadily increasing in many parts of the world, suggesting that current public health interventions are not working well.

Unlike most other skin cancers ${ }^{1}$ the prognosis is fairly dismal, with an overall case fatality rate of about 20\% [Marks, 2000: 460]. However, as I'll discuss at some length later, this rate varies heavily depending upon features of the particular tumour in question. It is, in general, extremely hard to predict just which tumours are likely to do well, and those which are likely to lead to the death of the patient. ${ }^{2}$

What causes melanoma? The broadest answer, found in material such as health promotion literature, is that exposure to UV radiation - primarily sun exposure during tanning - is the main cause of melanoma. For instance, the Cancer Council Australian website states:

"Melanoma risk increases with exposure to UV radiation, particularly with episodes of sunburn, Also at risk are people who have: increased numbers of unusual moles (dysplastic naevi); depressed immune systems; a family history (in 10\%, some having mutations in genes CDKN2A and CDK4); fair skin and; had a previous melanoma."

[http://www.cancer.org.au/aboutcancer/cancertypes/melanoma.htm]

That suggests that, for most 'normal' people developing melanoma, it is sun exposure that is doing most of the causing. But there's also a suggestion of a more complex situation. A more nuanced reading of the causes also largely supports the important causal role of sunlight. For instance, in a review of the epidemiology of the tumour [Marks, 2000: 461-3] the combined influence of constitutional and environmental factors is discussed. Constitutional risk factors include fair skin, number of moles (melanocytic naevi), tendency to freckle, family history of melanoma or atypical naevi, Xeroderma pigmentosa and increasing age. The main environmental factor is sun exposure, with no clear pattern of risk from other purported risk factors, including sunlamp use (either UVA 320-400nm or UVB 290-320nm), smoking, hair dyes, fluorescent lighting, HRT or stress. Sunscreen use seems to positively correlate with melanoma risk, but the research is heavily confounded and controversial [see, e.g. Garland et al, 1992; Williams, 2003: 287ff]. The author notes: "...there have

\footnotetext{
1 "...it has also become evident that melanoma is distinct from other tumours with regard to its cellular origin and pathophysiological mechanisms." [Kuphal and Bosserhoff, 2009]

2 "Cutaneous melanoma is a most unpredictable lesion. The marked variation in prognosis is probably a function of many variables...." [Breslow, 1970: 902]
} 
been no studies in humans, to date, that clearly demonstrate that the use of sunscreens alone can reduce the risk of melanoma." [Marks, 2000: 463].

The causal relationship between melanoma and sun exposure is interestingly complex. While outdoor workers have very high total sun exposure, and correspondingly high incidence of nonmelanoma skin cancers, they have much lower incidence of melanoma than indoor workers. This suggests that episodic sun exposure is more important than continual. The ratio between types of sun exposure also seems to determine the type of melanoma that develops - that is, different types of melanoma appear to be caused by different forms of sun exposure.

This complex situation is found in the research literature. There seem to be a group of complex responses to sun exposure. As Curtin et al [2005] point out, there appear to be different mutations in different melanoma types. Added to the recent reviews of the genetic pathogenesis of the condition [Kuphal and Bosserhoff, 2009, for example], it appears there a complex set of environmental and constitutional factors are required to cause melanoma. These do not necessarily correlate well with the recorded types of sun exposure - e.g. cutaneous non-sun damage melanoma show high frequencies of BRAF/N-RAS mutations while other forms of non-sun damage melanoma, notably acral and mucosal, tend to show mutations in the CDK4 (cyclin-dependent kinase 4)or CCND1 (cyclin D1) pathways. All this suggests that the mucosal, chronic sun exposure, non-sun and acral forms of melanoma have distinct aetiological and pathological features, and much ongoing work is directed in figuring out how deep the divisions between subtypes of melanoma go.

This difference in the nature of causal factors as found in the different literatures is, I suppose, to be expected. We would not expect general health promotion advice, broad-scope research reviews and cutting-edge laboratory research to approach their audiences in the same way. But I suspect that in this particular case, this conflict is a symptom of a more profound problem grounded in the nature of melanoma classification. These difficulties have, in part, led to difficulties with determining, exploiting and explaining causation. This affects research into both aetiology and management. I also suspect that the business of classification itself has been damaged by upstream, earlier attempts at classification and its impact on aetiological and therapeutic research. This circularity means that it is not easy to cleanly unpick this problem. But I hope that I can lay out its broad structure in the following pages.

\section{§2: The history of melanoma, and its classification}

The initial modern descriptions of the disease dates from the work of Laennec [1806; see also Denkler and Johnson, 1999], with the first English description of the tumour in 1820 by Norris [Norris, 1820; see also Handley, 1907a: 933]. The term 'melanoma' was first used by Carswell [1838]. We have good evidence, though, that people were developing melanoma long before the nineteenth century. For example pigmented skin tumours were described in the Hippocratic corpus during the 5th CBE, and evidence of metastatic melanoma has also been found in human remains dating from a similar period [Urteaga and Pack, 1966].

Nineteenth century researchers did a very clear job of describing the clinical features of the disease. For instance, Rolleston [1899] presents a detailed description of a case of metastatic melanoma (more correctly 'melanotic sarcoma'):

"In this case the diagnosis was perfectly easy, as the history of removal of a melanotic sarcoma from the right eye left very little doubt that the enlargement and nodular condition of the liver was due to recurrence in that organ. As a proof that this diagnosis was correct the presence of melanin in the 
urine was all that was necessary; but another proof which would if present have further clinched the diagnosis was the presence of secondary melanotic nodules in the skin.

The occurrence of secondary melanotic sarcoma in the liver is well known and is such a striking morbid lesion that once seen-and all museums have specimens illustrating it-it is never forgotten."

[Rolleston, 1899: 1273]

So the clinical appearance, natural history, progression, complications and diagnostic tests of the disease are so familiar to the author - and hopefully his audience in a general medical journal - that they need no justification. But when he considers the aetiology of the condition, there are strikingly differences from the modern conception. For instance, the author suggests that most of these tumours arise in the eye, and originate rarely in the skin. This is the reverse of current opinion. There was a further debate on the origins of melanoma:

"From the unsettled state of opinion at the present time some authors prefer to speak of the pigmented tumours... as "melanomas" or simply as "pigmented tumours, " without labelling them as "sarcoma" or "carcinoma." In the meanwhile we should judge each case on its microscopic merits and not assume that a pigmented tumour is necessarily sarcomatous. It is not, as far as can be seen at present, a matter of any great clinical importance what the exact structure of a malignant melanoma is, though more careful classification in the future might be expected to show that there was some difference in the rapidity of generalisation."

[Rolleston, 1899: 1274]

This was controversial. Clarkson [1899] argues that Rolleston misses the significance of the causation of the tumour:

"One cannot, however, help being struck by the fact that though he has carefully summarised the present state of our knowledge of the subject of primary pigmented tumours the most important links of the chain are yet wanting and really very little is definitely known of their true nature."

[Clarkson, 1899]

In general, the clinical syndrome was well described during the second half of the nineteenth century, while the pathology, aetiology and classification of the tumour remained controversial well into the twentieth. For instance, the sarcomata/carcinomata question raised above persisted into the mid-twentieth century as a therapeutic and aetiological dispute [Anon., 1939]. There were also questions about the relationship between pigmented moles and melanoma [Handley, 1907b], pathological versus clinical classification [Anon., 1939] and features of early malignancy in moles [Grey-Turner, 1939].

So how have melanomas been classified ${ }^{3}$ We are generally accustomed to think of melanoma as a single pathological entity, with a number of possible clinical manifestations which share a broadly common aetiology. Taken from the mid-1960s, where the clinopathological entity of melanoma really began to assume its modern form, there have been five main forms of classification:

1. No classification. For example, Ackerman [1980] denies the possibility of a useful classification of melanoma

2. Classification by morphology of primary tumour

I. Morphological classification by gross appearance. Examples include Clark et al, 1969 (Clark II)

II. Morphological classification by tumour microstructure or invasion. For example Clark et al, 1969 (Clark I) and Breslow, 1970.

3 I've included an appendix giving some example classifications in detail. 
III. Morphological classification by both macro- and micro-features. For instance McGovern et al, 1973

3. Classification by site of origin. For instance, ICD-10.

4. Classification by combination of features of both primary tumour and metastases. For example, AJCC, 2002 [Greene et al. 2006], which is a combination of the Clark level, ulceration and tumour thickness for the primary tumour, plus nodal or distant metastases.

5. Classification by aetiological classification. I mean this in the modern sense, generally referring to the presence of causal genetic mutations, rather than the carcinoma/sarcoma debate. Example - Curtin et al, 2005.

Two short examples:

First, the cutaneous / acral / nodular / lentigo system, an example of classification by gross morphology, based on Clark II (see appendix). This derives from Clark et al [1969] and McGovern [1973]. Tumours may occur on the skin - meaning face, trunk, back and legs in particular (cutaneous). They may occur on the palms, soles and under the nails (acral). They may be nodular meaning that the invasive tumour forms a lump on the skin, rather than appearing to spread through normal skin. Or they may be lentigo maligna - a flattened, non-invasive form of melanoma.

Second, the mucosal / acral / cutaneous / cutaneous with signs of chronic sun damage system. Again, this is a classification by gross morphology. Annoyingly, I've been unable to find a good citation for the origin of this system. But it's in widespread use - for instance, Curtin et al, 2005 initially partition their trial population using it. This sub-classifies cutaneous tumours into those that arising from skin with signs of chronic sun damage, and those that do not. It also adds the classification of mucosal melanoma, which is a melanoma that originates on a mucosal surface such as inside the mouth.

At the outset, I think it's worth highlighting one major feature of melanoma classification - that these systems of classification have proven rather unstable. Newly introduced classifications have tended to be taken up and dropped from use very quickly. There are a couple of exceptions - the Clark level and the Breslow depth in particular - but the general impression is one of transience.

The later classifications tend to be more complex than the earlier ones - with exceptions such as the aetiological classifications, which at the moment tend only to pick out single (or very small numbers, anyway) of genetic changes as targets for dividing their classifications.

These classifications tend not to work very well. This applies not only to the primary purposes of classification in medicine - something that I'll discuss in the next section - but they are also fairly unsatisfactory in their ability to partition without question-begging. So, for example, there are many tumours that are not easy to cleanly classify using these systems. Further, some of the features used as classificatory markers appear suspiciously circular. For instance, dividing lentigo maligna from nodular melanoma seems to require the classifying clinician to make a call of judgement. Part of this problem may also be connected to the short lifespan of classifications - something noted by BenPorat et al [2006] with the introduction of AJCC in 2002.

I will also suggest that a common theme to these systems is the monistic tack taken by many of the authors. Useful past attempts at classification are not added to. Rather, they are replaced, or at best modified and subsumed into an altogether novel system. There are some apparent exceptions (e.g. Breslow thickness, Clark level). But here it is to be remembered that this does not constitute retention of the former system. Rather, this is the beneficent naming of a new subcomponent of the novel system of classification that shares some features with full-blown classificatory systems of the past. Take the example of the Breslow depth. This scheme originates in Breslow [et al, 1970] and 
persists in, for example, the AJCC 2002 system. But those retained features are not the same as the Breslow's original system of classification - the depths have changed (see appendix).

\section{§3: Types and purposes of classification}

What is all this classification for? In order to deal with that, I first need to distinguish general systems of classification from those used specifically for staging and grading tumours.

Staging is generally used as a tool to assess disease progression in a single case, or to easily select and describe trial populations. Staging systems are generally arranged along the TNM lines common to many types of malignant disease. This involves giving a numerical grade for $T$, the tumour stage (diameter or thickness, for example), for $\mathrm{N}$, the nature of the disease in lymph nodes (so 0 - no nodal involvement, 1 , some nodal involvement, 2 , regional nodal involvement) and for $M$, the degree, if any, of metastasis seen. The outcome of a classification can then be recorded as a three figures shorthand indication of the state of the disease. So a person might be T1NOMO, or T3N1M1. Groups of TxNxMx can then be aggregated to give a single figure staging result 0-IV (see appendix). This gives clinicians a useful shorthand tool to discuss the progression of a particular case of a tumour, or for researchers to pick trial subjects at a particular disease stage. The AJCC scheme is an example of a staging system.

But staging doesn't say very much about the cytological nature of the tumour itself. Grading, on the other hand, does. This is a specialist histopathological tool, which involves describing the appearance of tumour cells under the microscope. Relatively normal looking tumour cells are said to be low grade, while highly abnormal looking cells are high grade. In general terms, the higher the grade, the faster the tumour will grow. This is used for prognosis predictions and for therapy selection. One reason that I haven't given examples of grading systems is that the gross morphological classifications are often taken as simple indicators of tumour grade. For example, lentigo maligna tend to be lower grade than superficial spreading melanomas.

$I^{\prime} l l$ discuss how grades and stage fit with my suggestions about classification in general in $§ 5$. So much for stages and grades. But what of the other classification systems? What are they for?

I can see three main purposes, which partially overlap with those of stage and grade. The first is classification for epidemiological data-collection for the purposes of finding out melanoma aetiology. As we discussed above, different forms of melanoma appear to have different aetiologies, particularly with regard to the nature of sun exposure. Thus several classifications consider markers of sun exposure - such as primary tumour site or signs of sun-damage on surrounding skin - as important classificatory features. Thus the causal importance of sun exposure can be estimated from tumour type, given a classification that uses sun markers as classificatory features.

But this appears problematic in practice because of the habit of thinking about risk factors, ${ }^{4}$ rather than causes. Part of the importance of systems of classification in data-collection is to organise attempts to digest complex risk factors into specific causal factors. For instance, researchers have tried to isolate the specific bit of sun-bathing that results in melanoma, by for instance looking at melanoma rates in habitual users of artificially generated UV light. This sharpening of risk into causal factors has much in common with the literature on mechanisms, in particular that on mechanism construction [e.g. Craver, 2007] for instance opening 'black boxes' - figuring out the specific mechanistic steps that constitute an identified risk factor. I don't have the space to develop this

\footnotetext{
4 By which I mean something like blunt, general, complex behaviours or characteristics that correlate with the disease. So 'poverty' is often cited as a risk factor for many diseases. But this doesn't really tell you anything useful about causes.
} 
theme as I would like, but I might suggest that the factors identified by Marks [2000] are firmly in the risk factor camp, and have both complex natures (e.g. outdoor worker) and complex relationships with one another (e.g. freckling and sun exposure).

The second is for prediction, especially for prognostic purposes. So for instance metastatic tumours have a much worse prognosis in terms of survival than localised ones. Most of the classifications take some consideration of the extent of the tumour. This allows data collection about survival for a particular tumour spread, and thus decisions about prognosis can be made for the individual case. Staging systems are important examples of classifications designed to deal with prognosis.

The third is as a guide to therapy. Different types of tumour (not to mention different grades and stages) require different treatments. So this third purpose of classification is in some ways parasitic on data-collection and prognosis. But there are areas where it is an independent purpose. Take the case of specific targeted treatment - for instance, the use of imatinib in c-KIT - which I'll talk about in $\S 4$.

As can be seen from this short outline, the various tasks that classifications serve are intimately linked. The classification used to do aetiology will carry over into trials of treatment. From here, they will be used to assess prognosis and outcomes. Without some epistemically grounding, unhelpful features used to construct classifications may propagate endlessly. That is, I suspect our classifications may fail silently. Heterogeneity within treatment outcomes may not be apparent if we cannot describe what it is that distinguishes cases. Similarly, features that would be useful in classification may be neglected. See e.g. Anonymous, 1965 for an early description of the confusion that can result.

Some have argued for strongly unificatory classifications of melanoma - that is, to give up on intramelanoma classification altogether. I have to strongly disagree with this point. I think that the rewards of classification are worth all the conceptual and empirical bother. In fact, it appears to me that the (recorded) differences between types of melanoma have already suggested the possibility of a better classificatory system that those in wide-spread clinical use. That is a system of classification based around causes.

\section{§4: Therapeutics and causation}

How well do current classifications work? Not well. Current melanoma outcomes vary - by most of the systems of classification listed above. But in general they are not impressive. While about $80 \%$ of small, thin, localised melanomas can be cured surgically, by far the commonest outcome of metastatic disease is death. Overall, 5 year survival is about $5-10 \%$. I have no idea just how much these outcomes are influenced by classificatory confusion. But I'll give an example which demonstrates the plausibility of current classification causing harms.

Typically, expected improvements in outcomes are modest for new melanoma treatments. Trials of new agents are set up to typically detect single-figure movements in outcome markers. Very often these modest aims are not met. Take c-KIT mutation. This mutation is not found in intermittent-sunexposure melanoma. Only about $30 \%$ of (largely mucosal, acral and chronic-sun exposure) melanomas express c-KIT [Natali et al, 1992]. An agent that blocks the c-KIT pathway - imatinib - is widely available as a treatment for leukaemia. This agent has been shown to work in case reports [e.g. Hodi et al, 2008; Lutzky, Bauer and Bastian, 2008], and it seems likely that it will be successful in clinical trials as a treatment for this specific sub-set of the disease.

Yet imatinib has been previously ruled out as a useful therapeutic agent, with significant reported toxicity but no effect [Wyman et al, 2006]. But here classification rears. This study took patients 
without selecting by tumour genetics. As by far the majority of melanomas do not express C-KIT, it therefore seems likely that the negative results of this trials (survival or time to progression) can be explained in terms of classification - or, at least, that any definitively negative result must wait for trials looking at the population likely to benefit from the treatment. Put another way, it could well be the case that we have an instance of Simpson's paradox occurring here, and much different treatment outcomes for one study group if the data were partitioned by c-KIT status. As it is, any likely improvement due to imatinib is likely to be swallowed by the much larger group of c-KIT negative subjects who have effectively received placebo. We may, in this case, be missing out on a useful treatment just because the system of classification in use fails to cut at the most appropriate joints. Luckily for us, new trials of imatinib are currently in progress using just this methodology.

How can we evade this sort of situation? Rather than classifying on the grounds of plausible (or confirmed) risk factors, macroscopic tumour features, histology etc, I suggest that we should cut to the chase, and classify based on specific causes, as is already beginning to happen [Curtin et al, 2005] in practice. But how and why is this likely to help?

\section{§5: Exploiting causal relationships in the melanoma case}

"...once the driving genetic alterations of a melanoma are known and effective drugs are available to counteract them, strong and immediate responses can be expected."

[Lutzky, Bauer and Bastian, 2008: 493]

How does introducing a causal classification improve melanoma classification? First, we evade the circularity that occurs in current classifications. No longer would the only tests of classification systems be trials that rely on the same systems. Instead epidemiologic and therapeutic research could be supported by laboratory science in a more visible manner

We expect causal relationships to be more robust and generalisable than non-causal correlations. Thus, we should expect better treatments to become available, partly through improvements in testing them. ${ }^{5}$ If we can do our epidemiological research using a causal classification, we should expect our therapeutic trials that rely on the same causal features to test their new treatments to give more reliable results. That is to say that we should expect not to miss positive results (as potentially happens in the C-KIT case). We should expect similar benefits in prognosis research.

Finding causes is also likely to lead to benefits in epidemiology. Once some causal factors are understood, the current mess of interdependent, complex risk factors becomes more amenable to analysis.

Finally, the heuristic of 'hunt causes' is beneficial in itself, even if we do not actually discover specific causes. For one, a sense of dissatisfaction with risk factors suggests which areas we should focus our investigative efforts on. For another, failing systems of classification can no longer do so silently. In the current situation, most classifications of primary tumours have plenty of 'wiggle-room' - that is, we can force tumours into one or other category, even if they do not really belong there. This is generally talked about as operator dependency. But classifications based on specific, dichotomous causal factors are much less prone to this sort of creep. Instead, classificatory problems are highlighted, rather than glossed-over.

\footnotetext{
5 There might also be a direct benefit from thinking causally, in that we can develop specific targeted treatments more easily.
} 
But there are of course difficulties with this approach. For one, finding causal factors from risk factors is incredibly hard. Knowing, and being certain that you know, the significant, differencemaking cause even more so.

Some notion is required of specific causation so that we know that we are dealing with causal, as opposed to risk, factors. For instance, what is it that makes sun-bathing a risk factor, but exposure to a particular wavelength radiation at particular intensity for particular time a causal factor? Why was the nineteenth century debate over the sarcomatous or carcinomatous origin of melanoma not the sort of cause that we need to do this work? These are questions I cannot answer here, although I suspect that a strongly reductive view of causation is unlikely to be helpful here.

Finally, finding a cause does not guarantee good results. We cannot expect that finding a single cause will utterly revolutionise melanoma classifications. The same classifications are popular now, even though we know about c-KIT, BRAF and so on.

In conclusion, a demand for an instant shift to all-causal classifications is daft. Perhaps the answer, unlike many previous classifiers, is to be more pluralistic. A causal system is compatible with the useful features of classifications in use. In particular, staging and grading systems can - relatively easily - be adapted to employ causal factors, rather than risk factors or tumour features. This means that we have no need to rewire all existing classifications to introduce this new system - something that appears to have been a problem with previous attempts at classification. Causal classifications are highly compatible with pluralism.

Grading and staging fit well into a Dupré-type promiscuous realism. He suggests that multiple systems of classification may happily co-exist. Speaking of the tendency of distinct groups to classify the same objects differently: "...diverse groups of people require workable classifications that enable them to communicate among themselves and to members of other such groups, record information... and so on." [Dupré, 2001: 204].

But I don't think we can make the case that all these systems of classification would fit equally well into such a conception. I'd suggest that this does not mean that all these systems of classification are equally good, nor should we believe that each of them cuts nearest to the joints. In fact the variable nature of predictive success for these systems is startling. Given the empirical results of these systems of classification, the current arrangements suggest that we are not cutting nature's joints. Rather we are aimlessly hacking into a pulp.

I want to suggest that the classification based on causation within a pluralistic framework is the best way to proceed. Even if attempts to classify causally are unsuccessful, the strategy of attempting a causal classification is an excellent way to proceed to better healthcare outcomes. 


\section{Appendix: Examples of melanoma classifications}

\section{Clark et al, 1969}

This paper contains two systems for melanoma classification, which I've dubbed Clark I and II. Clark I is well-known, and still widely used - also known as the Clark level. But originally Clark II was the primary system of classification, with Clark I used to sub-classify within melanoma types.

\section{Clark I}

Five anatomical levels of deepest invasion. The lower the level, the worse the prognosis:

Level I. Melanoma confined to the epidermis (melanoma in situ)

Level II. Invasion to the basal layer epidermis

Level III. Invasion to the papillary dermis

Level IV. Invasion to the reticular dermis

Level V. Invasion to the subcutaneous fat

\section{Clark II}

This is a histogenetic classification into three (specific) types and one dust-bin:

1. Superficial spreading melanoma

2. Lentigo maligna

3. Nodular melanoma

4. Melanoma NOS (not otherwise specified)

This is still a common way of classifying melanoma clinically, with the addition of a fourth histogenetic type - acral - by McGovern et al, 1973. It also subsumes a lot of other attempts at classification e.g. Trapl's horizontal/vertical system [Trapl et al, 1966], the Petersen pigmented flare (Petersen et al, 1962) and Davis' pedunculated/polypoid classification [Clark et al, 1969; ref. to personal communication with Davis pp. 714].

This is the origin of classification in ICD9.

\section{Breslow, 1970}

This paper looked at the relationship between maximum tumour thickness, cross-sectional areas and depth of invasion in an attempt to form prognostic indicators, particularly of metastasis. It was the thickness of lesions that was the best prognostic indicator. Now known as Breslow depth, it is adapted to form part of other schemes - e.g. AJCC.
I. $\quad<0.76 \mathrm{~mm}$
II. $\quad 0.76-1.50 \mathrm{~mm}$
III. $\quad 1.51-2.25 \mathrm{~mm}$
IV. $\quad 2.26-3.00 \mathrm{~mm}$
V. $\quad>3.00 \mathrm{~mm}$ 
American Joint Committee on Cancer Staging System for Cutaneous Melanoma (AJCC) 1988, 1992, 2002 [Greene et al. 2006]

This is a combination scheme, primarily used for staging. It combines the features of the primary tumour (Clark level, Breslow depth and presence or absence of ulceration) with features of lymph nodes and distant metastases.

Tumours are classified as TxNxMx. Particular groups of TNM are then combined to give stage $0-I V$. For instance, stage lla is T2bNOMO, T2bNOMO, with a 5 year survival of $77-79 \%$.

T1. Thickness $\leq 1.0 \mathrm{~mm}$

a. no ulceration and Clark level II/III

b. ulceration or Clark level IV/V

T2. Thickness $1.01-2.0 \mathrm{~mm}$
a. no ulceration

b. ulceration

T3. Thickness $2.01-4.0 \mathrm{~mm}$
a. no ulceration

b. ulceration

T4. Thickness $>4.0 \mathrm{~mm}$
a. no ulceration
b. ulceration

NO. No evidence of nodal metastasis

N1. 1 node

N2. 2-3 nodes
a. micrometastasis
b. macrometastasis

a. micrometastasis

b. macrometastasis

N3. 4 or more metastatic nodes, or matted nodes, or in-transit metastases/satellites and metastatic nodes

M0. No evidence of metastasis to distant tissues or organs

M1. Distant metastases

M1a. Distant skin, subcutaneous or nodal metastases

M1b. Lung metastases

M1c. All other visceral metastases or any distant metastases

C43 Malignant melanoma of skin

C43.0 Malignant melanoma of lip

C43.1 Malignant melanoma of eyelid, including canthus

C43.2 Malignant melanoma of ear and external auricular canal

C43.3 Malignant melanoma of other and unspecified parts of face

C43.4 Malignant melanoma of scalp and neck

C43.5 Malignant melanoma of trunk

C43.6 Malignant melanoma of upper limb, including shoulder

C43.7 Malignant melanoma of lower limb, including hip

C43.8 Overlapping malignant melanoma of skin

C43.9 Malignant melanoma of skin, unspecified 


\section{References}

Ackerman, A.B. 1980. “Malignant Melanoma: A Unifying Concept," Human Pathology. 11(6): 591-5.

Anonymous, 1939. “Melanoma," The Lancet. 234(6070): 1376.

Anonymous, 1965. "Malignant Melanomas of the Skin," The Lancet. 286(7423): 1171-2.

Ben-Porat, L. et al. 2006. "Estimates of Stage-specific Survival are Altered by Changes in the 2002 American Joint Committee on Cancer Staging System for Melanoma," Cancer. 106(1): 163-71.

Breslow, A. 1970. "Thickness, Cross-sectional Areas and Depth of Invasion in the Prognosis of Cutaneous Melanoma," Annals of Surgery. 172(5): 902-8.

Carswell, R. 1838. Pathological Anatomy: Part 9, Melanoma. London: Longman.

Clark, W.H. et al. 1969. "The Histogenesis and Biologic Behaviour of Primary Human Malignant Melanomas of the Skin," Cancer Research. 29(3): 705-27.

Clarkson, G.A. 1899. “Malignant Pigmented Tumours," The Lancet. 153(3951): 1392.

Craver, C.F. 2007. Explaining the Brain: Mechanisms and the Mosaic Unity of Neuroscience. Oxford: Clarendon Press.

Curtin, J.A. et al. 2005. "Distinct Sets of Genetic Alterations in Melanoma," New England Journal of Medicine. 353(20): 2135-47.

Denkler, K. and Johnson, J. 1999. "A Lost Piece of Melanoma History," Plastic and Reconstructive Surgery. 104(7): 2149-53.

Garland, C.F., Garland, F.C. and Gorham, E.D. 1992. "Could Sunscreens Increase Melanoma Risk?," American Journal of Public Health. 82(4): 614-5.

Greene, F.L. et al. 2006. AJCC Cancer Staging Atlas. $6^{\text {th }}$ edition. Berlin: Springer.

Grey Turner. 1939. Transactions of the St. John's Hospital Dermatological Society. London: 93ff.

Handley, W.S. 1907a. "The Bunterian Lectures on the Pathology of Melanotic Growths in Relation to Their Operative Treatment," The Lancet. 169(4362): 927-33.

Handley, W.S. 1907b. "The Bunterian Lectures on the Pathology of Melanotic Growths in Relation to Their Operative Treatment," The Lancet. 169(4363): 996-1003.

Hodi, F.S. et al. 2008. "Major Response to Imatinib Mesylate in KIT-mutated Melanoma," Journal of Clinical Oncology. 26(12): 2046-51.

ICD-10. 2007. International Statistical Classification of Diseases and Related Health Problems, $10^{\text {th }}$ Revision. 2007 version. Geneva: World Health Organization. Available online at http://apps.who.int/classifications/apps/icd/icd10online/ 
Kuphal, S. and Bosserhoff, A. 2009. "Recent Progress in Understanding the Pathology of Malignant Melanoma," The Journal of Pathology. Article proof, DOI: 10.1002/path.2617.

Laennec, R.T.H. 1806. "Sur les melanoses," Bulletin de la Faculte de Medecine de Paris. 1: 24-6.

Lutzky, J., Bauer, J. and Bastian, B.C. 2008. "Dose-dependent, Complete Response to Imatinib of a Metastatic Mucosal Melanoma with a K642E KIT Mutation," Pigment Cell \& Melanoma Research. 21(4): 492-3.

Marks, R. 2000. “Epidemiology of melanoma," Clinical \& Experimental Dermatology. 25(6): 459-63.

McGovern, V.J. et al. 1973. "The Classification of Malignant Melanoma and its Histologic Reporting," Cancer. 32(6): 1446-57.

Natali, P.G. et al. 1992. "Progression of Human Cutaneous Melanoma is Associated with Loss of Expression of c-kit Proto-oncogene Receptor," International Journal of Cancer. 52(2): 197-201.

Norris, W. 1820. "A Case of Fungoid Disease," Edinburgh Medical and Surgical Journal. 16: 562-5.

Petersen, N. C., Bodenham, D. C., and Lloyd, 0. C. 1962. "Malignant Melanomas of the Skin," British Journal of Plastic Surgery. 15: 49-94; 97-116.

Rolleston, H. 1899. "A Clinical Lecture on Secondary Melanotic Sarcoma of the Liver," The Lancet. 153(3950): 1270-5.

Trapl, J., Paleček, L., Ebel, J. and Kučera, M. 1966. Tentative New Classification of Melanoma of the Skin. Acta Dermato-Venereologica. 46: 443-6.

Urteaga, O. and Pack, G.T. 1966. “On the Antiquity of Melanoma," Cancer. 19(5): 607-10.

Williams, H.C. 2003. Evidence-based Dermatology. London: BMJ Books.

Wyman, K. et al. 2006. "Multicenter Phase II Trial of High-dose Imatinib Mesylate in Metastatic Melanoma: Significant Toxicity with no Clinical Efficacy," Cancer. 106(9): 2005-11. 